\title{
VIII.
}

\section{Ueber tropische Leberabscesse und ihr Verhältniss zur Dysenterie.}

\author{
Von Dr. Kartulis, \\ Arzt im Regierungshospital von Alezandrien.
}

(Hierzu Taf. VII.)

Die Kenntniss der tropischen Leberabscesse hat in den letzten Jahren keine besondere Förderung erfahren. Die darüber herrschenden Anschaunngen lauten so widersprechend, dass einige ermuthigende Ergebnisse meiner Untersuchungen mich zur neven Erörterung der Frage veranlassen.

In der Literatur findet man eine grosse Anzahl von Theorien, die jedoch die Pathogenese des Leberabscesses nicht zu lösen im Stande sind. Es sei mir hier gestattet, einige der wichtigsten davon wiederzugeben.

Es waren besonders französische Aerzte, welche zuerst den Einfluss der Malaria auf die Entstehung der tropischen Leberabscesse betont haben (Haspel, Foiret, Olmeta u. A.). Spätere Beobachtungen lehrten aber, dass tropische Leberabscesse ebenso oft auch in malariafreien Orten, wie Chile, theilweise auch Aegypten, vorkommen können, wie dagegen dieselben seltener in Gegenden wie Madras, Niederbengalen u. a. auftreten, wo Malaria endemisch herrscht ${ }^{1}$ ). Andere Forscher behaupten, dass schädliche Nahrungs- und Genussmittel bezw. Alcoholismus als Ursache der tropischen Leberabscesse anzusehen sind. Sachs") besonders begründet letztere Theorie also: "was der Alkohol für die chronisch-entzündlichen Prozesse der Leber in kälteren Ländern ist, das scheint er für die acute Hepatitis der heissen Klimate zu sein".

1) Vergl. Hirsch, Hist. Geogr. u. Path. III. Abth. 278.

2) Ueber die Hepatitis der heissen Länder. v. Langenbeck's Archiv Bd. XIX. 


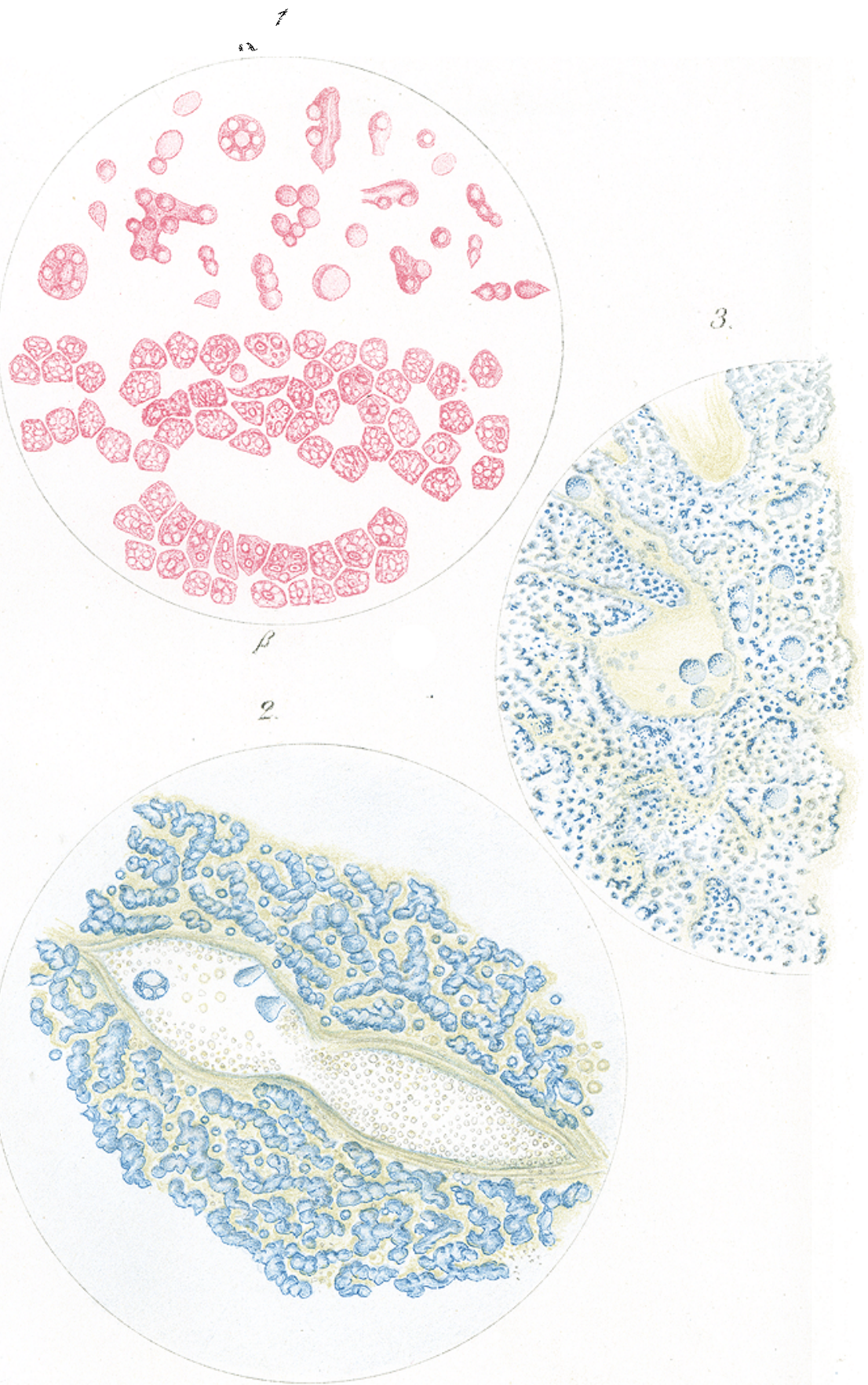


Larrey, welcher den französischen Feldzug nach Aegypten mit Napoleon mitmachte, hat verschiedenartige Entstehungsursachen des Loberabscesses angegeben. Insbesondere waren aber Strapazen, die grosse Hitze, Abusus von Alkohol, das Aufhören des Schwitzens und die Diarrhöe, die Anlass zu Leberabscessen gaben. Den Zusammenhang der Dysenterie mit dem Leberabscess soll Galenus schon gewusst habes. Im vorigen Jahrhundert sind es Lind, Petit und Pringl gewesen, welche dieser Meinung waren. Annesley (1828) hielt Ruhr and Leberabscess nicht als zwei verschiedene Erkrankungen, sondern beide zusammen als Folge einer Noxe. Mit anderen Worten nahm er an, dass bei der dysenterischen Hepatitis es geschieht, dass entweder die Dysenterie oder die Leberentzündung den Anfang machen. Anderes $\mathrm{Nal}$ beginnen alle beiden Symptome gleichzeitig. Bristow (1858) vertritt eine ähnliche Theorie, behauptend, dass die Leberabscesse nicht als Folge einer Darmverschwärung, die Dysenterie auch nicht eines Leberabscesses betrachtet werden müssèn, sondern dass eine gemeine Ursache bei einem Individuum Leberabscess, bei anderen Dysenterie und bei einem dritten beides hervorrufen kann.

Budd war einer der ersten, der den Leberabscoss als die Folge der dysenterischen Zerfallstoffe der Darmgeschwüre anerkannt hat.

Kiener und Kelschin ${ }^{1}$ endlich, Anhänger der Theorie von Annesley, äussern sich folgendermaassen: „il est plus commun de voir les symptomes de l'une alterer avec ceux de l'autre; c'est tantôt la dysenterie, tantôt l'hépatite qui ouvre la scène, et l'apparition de l'une est en général l'occasion, le signal d'une détente dans l'autre" und "la nature dysentérique de l'hépatite se trouve tout aussi bien établie par la dysenterie qui suit que par celle qui précède le développoment des lésions du foie".

Andere Forscher, wie Morehead und Murchinson, messen die Coincidenz zwischen beiden Krankheiten nur dem Zufall bei.

Dass der tropische Leberabscess nicht immer das Resultat der Dysenterie ist, wird noch unten besprochen werden, dass aber die Dysenterie sehr häufig Anlass zu der eitrigen

1) De la nature de l'hépatite suppurée de pays chauds. Archives gẻnérales de médécine. Sept. 1888. 
I deberentzündung giebt, dafür sprechen nicbt nur die Erfahrungen der meisten Beobachter, sondern auch vorzugsweise die Ergebnisse dieser Mittheilung. Die Statistik zeigt ebenfalls zur Genüge, dass die Mehrzahl der Leberabscesse nach Dysenterie entsteht. Nach Kiener's und Kelsch's Angaben kamen bei 314 Leberabscessen 268, die dysenterischen Ursprungs waren $(85,35$ pCt.). Nach einer Zusammenstellung von Hirsch wurden 19,2 pCt. Leberabscesse bei tödtlich verlaufenen Ruhrfällen angetroffen (2377 tödtliche Ruhrfälle, 457 Leberabscesse). Sir Joseph Fayrer ${ }^{1}$ ) hält 18 pCt. Leberabscesse bei Dysenterie als eine sehr wahrscheinliche Procentzahl. Von über 500 Leberabscessen, welche ich bis jetzt beobachtet habe, waren 55 bis 60 pCt. dysenterischer Natur. Ich besitze leider nicht mehr die mit grosser Mühe gesammelten Einzelheiten dieser Fälle, verfüge aber über eine Reihe von 33 Fällen, welche zum grössten Theil im hiesigen Regierungshospital beobachtet wurden. Die grösste Zahl dieser Fälle sind auch bakteriologiseh, wie auch mikroskopisch untersucht worden. Von diesen Fällen sind 22 Leberabscesse nach Dysenterie entstanden, die übrigen 11 mussten als sog. idiopathische Leberabscesse aufgefasst werden. Man stösst oft auf Fälle, wo während des Lebens eine Darmerkrankung in Abrede gestellt wurde und später die Section dysenterische Darmgeschwüre gezeigt hat (vergl. Fälle 3 und 4). In einer frïheren Mittheilung (dieses Archiv. 1886. Bd. 108) wurde.von mir eingehend ein a möboider Organismus beschrieben, dessen specifisch pathogene Eigenschaft für die ägyptische bezw. tropische Dysenterie dort besonders betont wurde. Bei Fortsetzung meiner Untersuchungen fand ich später dieses Protozoon in den Schnittpräparaten von mehreven nach Dysenterie erfolgten Leberabscessen. Wenn diese Befunde beziehentlich des Zusammenhanges beider Erkrankungen vieles aufklärten, so suchte ich die A möben im lebenden Zustande, wie es in den dysenterischen Stuhlausleerungen der Fall war, im Leberabscessinhalt anzutreffen. Ich kam bald auf einen geeigneten, nach Dysenterie erfolgten Leberabscessfall, wo sich die Amöben in der That am Leben im. Abscesseiter nachweisen liessen ${ }^{2}$ ). Es handelte sich (Fall 1)

1) Tropical dysentery. London 1881.

$\left.{ }^{2}\right)$ Vergl. Centralbl. d. Bakteriologie u. Parasitenkunde. 1887. Bd.II. No.25. 
um einen 20jährigen Araber, der seit 3 Monaten an Dysenterie litt und mit Leberabscesssymptomen im Regierungshospital aufgenommen wurde. Pat. erlag bald nach Eröffnung des Abscesses und bei der Obduction wurden zwei Leberabscesse gefunden, deren Eiter fast eine Amöbenreincultur darbot. Alsbald bestätigte sich dieser Befund bei allen von mir untersuchten dysenterischen Leberabscessen, während dagegen die Amöben bei allen Leberabscessen nicht dysenterischen Ursprungs fehlten. Die Amöben sind bis jetzt von R. Koch (in den Schnittpräparaten dysenterischer Darmgeschwüre, sowie auch in einem nach Dysenterie entstandenen Leberabscess) ${ }^{1}$ ) und von mir gefunden worden. Später fand $\mathrm{Hlava}^{2}$ ) in 60 Fällen von Dysenterie, die im Irrenhause von Prag vorkamen, die Amöben im Darminhalte. Vielleicht gehört hierher auch ein Fall von Lö $\mathrm{sch}^{3}$ ), der zuerst ähnliche Parasiten bei einem Dysenteriefall in St. Petersburg beobachtete.

Bis jetzt wird die Bedeutung der Amöben für die Dysenterie, bezw. Leberabscesse, so weit mir die Literatur zugänglich ist, entweder als ein zufälliger Befund erwähnt, oder auch ihnen die pathogene Eigenschaft abgesprochen. Baumgarten in seinen letzten Jahresberichten ${ }^{4}$ ) verlangt mit Recht die Pathogonose der Amöben durch Reincultur und Impfversuch zu erhärten. Wenn wir deu Ausführungen von Lösch und Hlava glauben wollen, so gelang beiden der Impfversuch. Ersterer spritzte amöbenhaltige Stühle in den Darm von vier Hunden ein. Einer derselben erkrankte und in seinen Ausleerungen fanden sich die Amöben wieder. Das Thier wurde getödtet und die Schleimhaut zeigte sich entzündet mit oberfiächlichen Geschwüren. Hlava erreichte ähnliche Resultate bei jungen Katzen. Er soll auch die Amöben rein gezüchtet und damit ebenfalls junge Katzen inficirt haben. Da Hlava's diesbezügliche in Aussicht gestellte ausführliche Veröffentlichung bis jetzt nicht erfolgt ist, so erlaube ich mir meine leider nicht sehr ermuthigenden Er-

1) Centralblatt der Bakt. und Parasitenk., a. a. 0.

2) Ebendaselbst. 1887. No. 18.

3) Dieses Archiv. 1875. Bd. 65.

4) Ueber die Fortschitte in der Lehre von der pathogen. Mikrogen. 1886 u. 1887. 


\section{1}

gebnisse zu erwähnen. Mit amöbenhaltigen Dysenteriestühlen gelang es mir bis jetzt noch nicht, Affen, Hunde, Katzen, Meerschweinchen und Kaninchen dysenterisch zu machen. Amöben lassen sich nicht, wie Bakterien, rein züchten. Im alkalisch reagirenden und steril gemachten Kaninchenmist wachsen die Amöben bei gewöhnlicher, sowie bei Körpertemperatur mit anderen Bakterien gemengt. Im sterilen Wasser, dem etwas Fleischbrühe oder Blutserum zugefügt wurde, gedeihen auch die Amöben. Nach der dritten oder vierten Umzüchtung sterben aber die Thiere $a b$, die Zucht ist auch nie rein gelungen. Derartige Culturen wurden gleichfalls verschiedenen Thieren in's Rectum eingespritzt. Der Erfolg aber war, dass nur zwei Hunde an Durchfall erkrankten. In ihren weissfarbigen Ausleerungen fanden sich wieder Amöben, die Thiere erholten sich jedoch bald von ihrer Diarrhöe. Trotz dieses fast negativen Thierversuches halte ich aufrecht, dass unsere Amöben für die Dysenterie (wenigstens diejenige Aegyptens) und die dysenterischen Leberabscesse als Ursache zu betrachten sind. In mehreren Fällen von Dysenterie, sowie auch von Leberabscessen, die von mir bakteriologisch erforscht wurden, vermochte ich in keinem Fall einen bestimmten Mikroorganismus vorwiegend anzutreffen. Gleichfalls liessen sich in den Darmschnittpräparaten verschiedenartige Mikroorganismen nachweisen. Chantemesse und $\mathrm{Vidal}^{1}$ ), welche in Cornil's Laboratorium 5 Fälle von Dysenterie untersuchten (1 Fall aus Tonkin, 4 aus Senegal und Cayenne) züchteten aus dem dysenterischen Darmschleim einen Bacillus, der anf Meerschweinchen verimpft, Darmentzündung hervorrief. Die geringe Zahl aber der untersuchten Fälle, sowie die angegebenen Veränderungen der Versuchsthiere, genügen nicht, wie ich glaube, die Aetiologie der Ruhr zu lösen. Wie es scheint, wussten diese Forscher von unseren Arbeiten nichts, da dort keine Erwähnung über einen Amöbenbefund gemacht wird. Ich will damit jedoch nicht behaupten, dass die Crsache von allen Klimatendysenterien bezw. Leberabscessen den Amöben zuzuschreiben ist. Ich will vielmehr auch zugeben, dass andere Mikroben Darmentzündung und Verschwärung verursachen können. Hervorheben muss ich aber, dass für die Dysenterie in

1) Semain médicale. 18. April 1888. 
Aegypten, Indien (Koch und ich), Sudan (ich) und für diejenigen in einigen Orten Europas vielleicht (Loesch in St. Petersburg und Hlava in Prag) die Amöben festgestellt wurden. Die Constatirung dieser Parasiten von mir bei mehr als 500 Fällen von Dysenterie, sowie bei allen dysenterischen Leberabscessen, ihr Fehlen andererseits bei anderweitigen Krankheiten, veranlassten mich, dieselben als die wirkliche Ursache der erwähnten Erkrankungen anzusehen.

Abgesehen von den klassisch pyämischen oder metastatisehen Leberabscessen, die auch in den kaiten Klimaten nicht selten sind, gewährt die Eintheilung in idiopathische und dysenterische Leberabscesse nach meinem Dafürhalten einen klaren Einblick in die Lehre derselben.

\section{Tropische Leberabscesse.}

1. Sog. idiopathische. Sie entstehen höchst wahrschein. lich durch Mikrobeninfection, welche aus dem Gastrointestinaltractus in die Leber gelangen. Als prädisponirende Momente können Malaria, Alcoholismus, Erkältung u. s. w. angenommen werden.

2. Dysenterische Leberabscesse. Einwanderung von mikrobenhaltigen Amöben von dem Darmgeschwür durch das Pfortadersystem in die Leber.

Idiopathische Leberabscesse. Da Zweck dieser Mit. theilung eigentlich die Entstehung der dysenterischen Leberabscesse ist, so werden die idiopathischen nur kurz erwähnt.

Es ist eine schon bekannte Thatsache, dass in den Tropen die Leber der fremden Bevölkerung in steter Blatüberfüllung sich befindet. Es ist ebenso leicht erklärlich, dass bei den Fremden durch ungeeignete Nahrungs. und Genussmittel Circulationsstörungen im Bereich besonders des Pfortadersystems leicht stattfinden kömen. Da die Stoffwechselphysiologio bei den verschiedenen Rassen in den Tropen noch wenig bekannt ist, so erklärt man vorläufig diese Zustände durch die dauernd hohe Temperatur und den schweren Luftdruck, welcher auf die Athmung ausgeübt wird.

Haspel') erwähnt von Algier, dass après un sejour pro-

1) Hirsch, Op. cit. p. 268. 
longé dans ce pays il n'est pas rare de voir le foie acquérir même dans un état sain un volume beaucoup plus considérable que celui, qu'il avait en France. Ueber Aegypten erwähnt Pruner ${ }^{1}$, "so jst dies noch mehr in Aegypten nnd hier weniger als in Arabien, Sennar und besonders Indien der Fall. Es zeichnet sich daher im afrikanischen Tropenlande beim Neger schon im relativ gesunden Zustande die Leber durch ihr Gewicht, ihre Grösse, ihr auffallend entwickeltes, körniges und dabei fettiges Gewebe aus.... Noch bemerken wir, dass bei gewissen fettreichen Personen, welche unmässig im Essen und Trinken zu sein pflegen, auf eine unglaubliche Art unter dem Einflusse der Hitze, die Leber so lange sie nicht tiefer leidet, einer periodischen Schwellung unterworfen ist, welche oft einige Tage sehr deutlich wird und dann wieder verschwindet".

Haymann's diesbezügliche Bemerkungen über das indische Erysipel lauten: „Es giebt eine Acclimations-Leberhypertrophie, die in den ersten Jahren nach der Einwanderung in die Tropenregion zum Ausbruche kommt, und dürften wenige Europäer davon befreit bleiben. In den an anderweitigen Krankheiten Verstorbenen findet man die Leber meistens beinahe um $\frac{1}{5}$ ihres Volumens vergrössert." Unsere Erfahrungen über diese Anlage von den fremden Rassen in den Tropen zur Leberhyperämie sind übereinstimmend mit den erwähnten Beobachtungen. Mit wenigen Ausnahmen pflegen die Weissen, welche zum ersten Mal das ägyptische Klima besuchen, über Leberbeschwerden zu klagen. Dieselben zeichnen sich besonders durch ein schweres Gefühl in dem rechten Hypochondrium und durch stechende Schmerzen daselbst aus. Der Schmerz wird empfindlicher durch tiefes Einathmen. Nicht selten gesellt sich hinzu ein Schultergefühl. Ueberhaupt man fühlt, dass man eine Leber besitzt. Diese Beschwerden können durch Erkältung, Darmkatarrh, oder durch anderweitige Ursachen verschlimmert werden. Die Leberschmerzen gestalten sich stärker und man findet bei der Percussion die Leber bedeutend vergrössert. Wenn die ursächlichen Momente nicht durch anpassende Diät und geeignete Behandlung behämpft werden, so führt der Prozess bis zur Abscessbildung.

1) Hirsch, Op. cit. p. 268.

2) Ibid. 
Viele Personen besitzen die Leber so empfindlich, dass sie bei jeder Erkältung, bezw. bei jedem Diätfehler daran erkranken. Viele Europäer acclimatisiren sich so schwer, dass ibnen der Aufenthalt in den Tropen unmöglich wird. Andere wieder ertragen diese leichten Leberentzündungen sehr lang, eine vollständige Immunität erlangt aber der Fremde nicht. Es ist nun einleuchtend, dass der Darm bei entzündeter Leber durch den beeinträchtigten Kreislauf im Pfortadersystem beeinflusst wird. Die dort gelegentlich befindlichen Mikroorganismen finden hierdurch ihren $W$ eg in die Leber. Einen directen Beweis dafür zu erbringen ist nicht möglich; man findet jeduch pathologisch-anatomisch im Darm die Schleimhaut entzïndet, das Epithel derselben theilweise abgestossen, die solitären Follikel breiter und geschwollen. Oft aber sind diese Veränderungen nicht sichtbar, was die Erklärungen der Absorption aus dem Darm, wenn noch andere Organläsionen fehlen, sehr erschwert. Sonst aber trifft man Veränderungen im Darme, die unerwartet waren. So z. B. fanden sich bei 2 sog. idiopathischen Leberabscessen Magengeschwüre, bei einem anderen Fall ein eingekeilter Olivenkern im Wurmfortsatz. Ein Umstand, dass im Intestinaltractos die primären Läsionen schwer nachweisbar sind, ist es noch, dass während des Vorhandenseius von Eiterhöhlen in der Leber passive Hyperämien im Darm vorkommen. Gleichfalls geht dadurch der Abfluss der Galle sehr mangelhaft von Statten. Es entstehen deshalb oft septische Prozesse im Darm, welche sehr oft zu profusen Diarrhöen führen. Die Darmschleimhaut bietet sodann derartige Veränderungen, welche die primären Läsionen verdecken können. Dass im Gastrointestinaltractus die Eingangspforten der pyogenen Mikroorganismen sich befinden, dafür sprechen ausser den erwähnten Beíunden im Magen und Blinddarmfortsatz, noch mehrere Unterleibsstörungen, die sich im Beginn der Krankheit durch schlechte Beschaffenheit der Zunge, hartnäckige Verstopfung oder Durchfall einstellen.

Wenn wir diese Fälle von idiopathischem Leberabscess näher betrachten, so finden wir, dass dieselben in der Mehrzahl Leute betreffen, die im reifen Alter sich befinden. Frauen werden gleichfalls, wenn auch viel seltener als Männer, davon befallen. Kinder scheinen überhaupt gegen Leberabscess immun 
zu sein. Wenigstens habe ich bis jetat keinen Leberabscess bei Kindern beobachtet.

In 11 idiopathischen Leberabscessen fanden sich bei der mikroskopischen Untersuchung des Eiters nur $2 \mathrm{mal}$ deutlich Mikroorganismon. Dieselben betrafen frische Abscesse, waren Mikrokokken und lagen in beiden Füllen innerhalb wie auch ausserhalb der Eiterzellen. In den übrigen Fällen waren keine Mikroben gefunden, was sonst bei nicht frischen Abscessen schon genügend bekannt ist. Zur Cultur wurde der Eiter 10mal verwerthet. Davon wuchsen in den Aussaaten (Platten von Peptongelatine, Agar-Agar, Rohrgläschen und Blutserum) in 4 Fällen der gelbe Eitercoccus, einmal der weisse Traubencoccus und in 4 Fällen blieben die Nährsubstrate steril. In den Schnittpräparaten der Leberabscesswandungen fanden sich Mikrokokken in 7 bei 8 untersuchten Fällen. Einmal waren überhaupt keine Mikroorganismen vorhanden. Die Mikroben waren besonders in den Capillaren, vorzugsweise zwischen Membrana pyogenica und intactem Lebergewebe vorhanden. In wenigen Fällen sassen die Mikroben in Zellen, die sich in dem Detritus der Membrana pyogenica befanden. Bei der Besprechung der dysenterischen Leberabscesse wird noch gezeigt werden, dass, abgesehen von der Anwesenheit der Amöben in den Jetzteren, kein wesentlicher pathologisch-anatomischer Unterschied zwischen beiden Leberabscessformen obwaltet.

Wenn ich jetzt zu den dysenterischen Leberabscessen übergehe, möchte ich noch mit einigen Worten das Verhältniss der Amöben zu den Darmverschwärungen erwähnen. An geeigneten dysenterischen Geschwüren findet man so zahlreiche Amöben, dass nicht nur das Geschwür von den Thierchen umschwärmt ist, dieselben sogar in alle Schichten der Darmwandungen eindringen. Es ist hesonders in den Capillaren der Submucosa, wo sich dieselben am meisten vorfinden. Einige davon überfüllte Gefässe zerreissen und es wird dadurch ein weiterer Substanzverlust erzeugt. Von den Capillaren aber wandern die Amöben durch die grösseren Aeste der Pfortaderwurzeln weiter in die Vena portarum und in die Leber. Selbstredend nehmen die Parasiten in ihren Leib nicht nur Mikroorganismen, sondern auch verschiedene Detritusmassen, sowie auch Blutkörperchen 
auf. In der Leber findet man dieselben alsbald in den Verästelungeu der Pfortenvene, entweder voll von Mikroorganismen, stäbchenähnlichen Gebilden, oder auch ganz frei davon. In den Detritusmassen des Leberabscesses, sowie auch in dem Abscessinhalt, nur selten Mikroben enthaltend, oft aber ganz leer. Ich erkläre dies aus dem Umstande, dass die lebenden Amöben durch die Bewegungen ihrer Pseudopodien, wenn dieselben grosse Mengen derartiger Gebilde in sich beherbergen, davon frei werden oder doch den Inhalt verdauen. Diese Annahme erklärt auch das Fehlen von Mikroorganismen in einigen Fällen. Dass die Amöben aber als Träger der letzteren, die den Anlass zu der Eiterung geben, dienen, muss trotzdem als sehr wahrscheinlich angenommen werden. Sie vermögen aber selbst durch die Zerreissung der Gefässchen eine Beschädigung des Gewebes hervorzurufen, die, wenn die Zahl der Amöben sehr zahlreich ist, eine bedeutende sein kann. Ob aber mikrobenfreie Amöben in der Leber zum Abscess führen können, halte ich für zweifelhaft, da auch Distomeneier und Embryonen (Bilharz) in die Leber eindringen, die Gefässe gleichfalls zerreissen, ohne jemals Abscesse hervorzurufen. Die stammen aber nicht aus dem Darm, sondern gehen direct von dem grossen Śtamm der Pfortenvene in die Leber, wo sie höchstens zu cirrhotischen Prozessen Anlass geben können, Leberabscesse wurden niemals nach Bilharz beobachtet $^{1}$ ).

Wären nur Darmgeschwüre die einzelnen Eingangspforten der Mikroorganismen, so könnte man erwarten, dass in den Tropen Tuberculose und Typhusgeschwüre auch zum Leberabscess führen könnten. Dies ist aber nicht der Fall. Beide Krankheiten, in Aegypten sehr verbreitet, gesellen sich nur äusserst selten zum Leberabscess und daun ist es fraglich, ob dort allein das Darmgeschwür daran die Schuld ist. Ich selbst sah zwei Fälle von Leberabscess bei Tuberculose mit Darmgeschwüren. Die mikroskopische Untersuchung aber des Abscesseiters wies

1) Kartulis, Ueber die pathologische Anatomie der Eier des Distomum haematobium (Bilharz) in den Unterleibsorganen. Dieses Archiv 1884. In 2 Fällen von Einwanderung von Askariden durch die Gallengänge in die Leber fand ich in der letzteren nur kleine unschriebene Eiterbeerde aber keinen richtigen Leberabscess. 
keine Tuberkelbacillen, sondern nur Amöben auf. Es scheint also, dass die Amöben durch ihre lebhaften Bewegungen eine gewebezerstörende Fähigkeit besitzen, die sich im Darm durch Verschwärung, in der Leber durch Zerreissung der Capillaren und Bluterguss in's Parenchym erkenntlich macht. Im letzteren Organ geschieht es, dass die Thierchen mit dem Blutstrom zwischen den Leberzellenreihen wühlen indem sie dieselben bei Seite abdrängen und drücken. Die die Amöben begleitenden Mikroorganismen spielen sodann die eigentliche Rolle bei der Eiternng.

Die Amöben in den Eiterhöhlen der Leber behalten ihre Lebensfähigkeit für längere Zeit, während Eiterzellen schon früher zu Detritus verfallen und Bakterien nicht mehr keimfähig sind. Wie lange die Thierchen dort am Leben bleiben, konnte ich nicht mit Sicherheit ermitteln. Während ich dieselben z. B. in zwei Leberabscessen, die wenigstens seit zwei Monaten bestanden, lebendig vorfand, fehlten sie bei einem dritten Fall, der seit drei Monaten bestand. In den Schnittpräparaten aber waren sie noch gut erhalten. Der Eiter des Abscesses war zu Detritus zerfallen, keine einzige Eiterzelle war intact. Einige CharcotLeyden'sche Krystalle waren noch sichtbar. Die Aussaaten des Eiters waren selbstredend nicht mehr keimfähig. In zwei anderen Fällen behielten die Amöben ihre Lebensfähigkeit in der Eiterblase nachdem schon die Darmgeschwäre seit 6-8 Wochen vernarbt waren. Die Amöben im Leberabscessgewebe verhalten sich folgendermaassen. In der Detritusmasse der Abscesswandungen findet man die Amöben isolirt, meistentheils rund oder auch in ovaler Form zwischen den amorphen Körnern. Ihre Grösse hier ist verschieden, gewöhnlich aber erscheinen sie hleiner als die im Eiter befindlichen Thiere. Am besten färbt man die Amöben im Gewebe mit Methylenblau bezw. Löffler'scher Lösung. Mit anderen Anilinfarben unterscheidet man die Amöben rom übrigen Gewebe schwer. Mit der blauen Farbe tingiren sie sich homogen, höchstens nur Vacuolen werden erkenntlich, während der Kern nicht sichtbar wird. Dagegen wird der Kern mit Alauncarmin intensiv, der Leib der Amöben aber leicht roth gefärbt. Nach innen findet man noch Amöben zwischen dem Detritus und dem intacten Gewebe in grösserer Anzahl zusammengereiht, auch jsolirt, umgeben entweder von ausgewanderten 
Leukocyten oder auch von ausgestossenen veränderten Leberzellen. Hier und da sind sie auch in kleinen intact gebliebenen Capillaren eingelagert. In einigen Fällen fanden sich die Thierchen beladen vou Bakterien, oft aber ist ihr Körper frei davon. In zwei Fïllen bildeten die Detritusmassen der Abscesswandungen Schwärme von bakterienhaltigen Amöben, die das Grundgewebe anfüllten und das Bild von Mikrobencolonien boten. Nach innen weiter die Thierchen verfolgend, findet man noch dieselben zwischen den Leberzellenreihen mit Lenkocyten und Blutkörperchen liegend. Hier sind sie bedeutend kleiner, und pur durch ihre eigenthümliche Gestalt, stärkere Färbbarkeit und manchmal durch ihre Vacuolen unterscheiden sie sich von anderen, besonders von ausgestossenen Leberzellen. Hier findet man $x \alpha \tau^{3} \dot{\varepsilon} \xi o x \eta^{\prime}$ in den Capillaren die Amöben. Das geschieht aber vorzugsweise in ganz frisch entstandenen Leberabscessen, während bei älteren Abscessen die Gefässe sehr erweitert sind und nur selten vereinzelte verkümmerte Amöbenleiber enthalten. Je nach dem Alter des Abscesses ist auch das Verhalten von Bakterien verschieden. In deo meisten Fällen findet man, wie es schon oben angedeutet wurde, gleichzeitig mit den Amöben noch verschiedenartige Mikroben, insbesondere aber Staphylokokken, welche die Parasiten begleiten. Die liegen auch meistentheils in Gefässen, oder auch findet man dieselben in Colonien zwischen dem Detritus, oder auch bei älteren Fällen in dem frisch gebildeten Bindegewebe eingeschlossen. In den Gefässchen, welche Amöben enthalten, waren merkwürdiger Weise in allen untersuchten Fällen keine Bakterien vorgefunden.

Bei älteren Leberabscessen sind die Amöben schwer zu erkennen, vorwiegend aber zeigen sie sich noch vereinzelt in kleinen umschriebenen Blutgerinsseln, welche vermuthlich durch Zerreissung kleiner Gefässe entstanden sind. Die Amöben fanden sich übrigens in zwei Fällen von dysenterischen Leberabscessen in kleinen Eiterheerden, welche sich in den Bauchdecken gebildet haben. Einmal war eine fistelähnliche Communication mit dem Leberabscess aufgefunden, im zweiten Falle fand sich eine solche nicht. Bei zwei anderen Fällen, wo der Eiter in die Pleura durchbrach, waren die Thierchen im Empyem auch nachweisbar. Ebenfalls in einem anderen Fall in einem secun- 
dären Lungenabscess. Drei Fälle von Hirnabscess, nach dysenterischen Leberabscessen orfolgt, sind in einer Zeit beobachtet, als mir noch die Amöben unbekannt waren.

Im Grossen und Ganzen ergiebt sich, dass die Amöben die Hauptrolle bei der Entstehung des dysenterischen Leberabseesses spielen, dass dieselben als Träger von Mikroorganismen dienen, welche letzteren den Eiterungsprozess vervollständigen.

Die bei dysenterischem Leberabscess vorkommenden Mikroorganismen scheinen vielen Gattungen anzugehören. Von 22 Fällen wurde der Eiter, der entweder durch Punction der Eiterhöhle oder bei der Obduction gewonnen wurde, 13mal zu Culturen verwendet. Das Ėrgebniss dieser Untersuchung war folgendes:

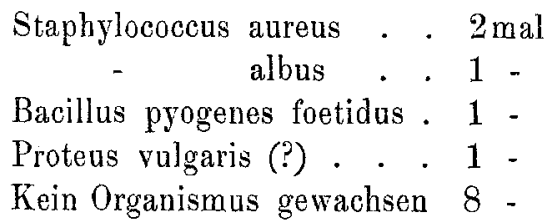

In den Schnittpräparaten der Leberabscesswandungen fanden sich manchmal verschiedenartige Organismen und dies war besonders bei Fällen, wo die Dysenterie, während des Vorhandenseins des Leberabscesses oder nach der Operation, noch bestand. In zwei Fällen fanden sich anscheinend Saprophyten, welche auf eine Verbindung des Abscesses mit den Bronchien, bezw. Veunreinigung der Abscesshöhle schliessen liess.

Kleine oberflächliche Leberabscesse, die, wie richtig Sir Joseph Fayrer betont, nicht richtige Leberabscesse, sondern Nekrose des Gewebes sind, kommen auch bei idiopathischen Abscessen vor. Die sind meistens oberflächlich und ihr Inhalt besteht nicht aus Eiterzellen, sondern aus nekrotischem Gewebe. Amöben und Bakterien wurden von mir hier ganz vermisst. In Schnittpräparaten erkennt man nur nekrotisches Gewebe, Zellen in körnigen Detritus zerfallen, und kleine Gefässe. Diese nekrotischen Inselchen entstehen nach meiner Ansicht nach Verschluss von weiteren Lumina von Capillaren durch thrombotische Prozesse. Wirkliche multiple Abscesse der Leber aber, die Eiter enthalten und kleine Höhlen bilden, werden wie die grösseren Abscesse gebildet. Derartige multiple Leberabscesse fand 
ich in meinen Fällen bei beiden Formen des tropischen Leberabscesses, entsprechen auch mikroskopisch im Kleinen dem schon bei grösseren Abscessen geschilderten Bilde.

Ich lasse hier einige Fälle von beiden Formen des tropischen Leberabscesses nachfolgen.

Fall 2. Ein 40jähriger Jude, seit mehreren Jahren in Aegypten, ist 14 Tage vor seiner Aufuahme im hiesigen Regierungshospital an Dysenterie erkrankt. Einige Tage darauf wurde er nach einem Schüttelfrost icterisch am ganzen Körper gefärbt. Die Krankheit wurde, da die Dysenterie keine Beschwerden mehr bot, für biliöses Typhoid gehalten. Schmerzen in der Lebergegend waren stark, die Anschwellung bedeutend. Patient fühlte sich noch am 40. Tage seiner Krankheit, als ich ihn sah, bei Kräften. Die icterische Farbe war vorbei, sonst war die Haut sehr anämisch. Dysenterische Ausleerungen begannen wieder und wurden so hartnäckig, dass Pat. äusserst entkräftet wurde und starb.

Bei der Obduction fand sich in der Mitte des rechten Leberlappens ein orangengrosser Abscess; der Eiter grünlich, sehr dickflüssig. Wandungen des Leberabscesses unregelmässig mit tiefen Ausbucbtungen. Die Leber selbst zeigt nichts Besonderes. Der ganze Dickdarm verschwärt. Ueberall zeigt sich die Schleimhaut desselben beschädigt. Die Geschwäre sind mit einander confluirt. Man unterscheidet aber einige derselben noch intact, umschrieben und verschiedener Grösse. Alle anderen Organe waren unverändert.

Im Leberabscessinhalt waren die Eiterkörperchen zerstört. Hier und da sah man einige Charcot'sche Krystalle. Weder Amöben noch Bakterien waren gefunden. Culturen des Eiters schlugen negativ ans.

Die mikroskopische Untersuchung des Darmes sowie anch der Leberabscesswandungen zeigte mit Sicherheit die Anwesenheit von gut, erhaltenen A möben. Im Ietzteren Organ waren die Amöben zahlreich zwischen den Detrituskörneben vorhanden. Nur hier und da merkt man in gant. kleinen Capillaren 1-4 Amöben. In den grösseren Gefässen fehlen die Amöben ganz.

Fall 3. Ein 30jähriger Mann aus Kreta lebt seit seinem 5. Lebensjahre in Aegypten. Derselbe soll niemals ernst erkrankt sein. In der letzten Zeit aber hat er öfters gehustet und ein paar Mal auch Blut gespuckt. 4 Wochen vor seiner Aufnabme in's Hospital ist derselbe an Dysenterie erkrankt. Nach eiver Woche sollen aber die dysenterischen Symptome auf einmal verschwunden sein, und seitdem füblte er sich noch kränklicher, fieberte und schwitzte Abends. Während dieser Zeit bemerkte er in seiner rechten Seite einen Tuwor wachsen, weshalb er auch in's Haspital Hülfe suchend kam. Bei der Aufnahme war Patient sehr mager und anämisch. Im rechten Hypochondrium, zwischen der Axillar- und Scapularlinie, ein faustgrosser Tumor, prall, deutlich fiuctuirend. Derselbe erweist sich durch Probepunction als ein Leberabscess. Es wurde sofort zur Operation geschritten und um den 
Abscess voliständig zu entleeren, resecirte ich die 9. Rippe. Die Abscesshöhle war faustgross, der Eiter rahmig. Eine Zeitlang schien Patient sich zu erbolen, die Eiterhöhle wurde bis zu einem Hühnerei verkleinert, als plötzlich eine hartnäckige Diarrhoe den Tod brachte.

Obdrction. Körper sebr abgemagert. Schleimhäute blass. Beide Lungenspitzen tuberculös infiltrirt und mit der Pleura stark verwachsen. Herz gross, weich, anscheinend fettig.

In der Mitte des rachten Leberlappens befindet sich eine hühnereigrosse Höhle, deren Wandungen $3 \mathrm{~cm}$ dick vernarbt sind. Die Höble enthält keinen Eiter. Von derselben führt ein Fistelgang zu einem nussgrossen Abseess, der in den Bauchmuskeln (Transv. abdomin. und Obliq. abdomin.) seinen Sitz hat. Dieser Eiterheerd communicirt wieder mit der äusseren Oeffnung und enthält noch Eiter. Milz gross $(7: 20 \mathrm{~cm})$, weist in ihrem Parencbym mehrere hämorrhagische Heerde auf. Auf der Oberfläche befindet sich ein erbsengrosser Abscess. Nieren gross und weich. In der Corticalis der rechten Niere ist ein kleiner oberflächlicher Abscess. Magen und Darm bieten nichts Besonderes als eine bedeutende Anämie der Schleimbaut. Dieselbe ist auch locker im Darm und zeigt deutlich die solitären Follikel. Im Dickdarm besonders sind keine Geschwüre vorhanden. Sogleich nach der Operation wurden ans dem entleerten Eiter mebrere Deckglaspräparate angefertigt. In allen fanden sicb sehr schöne Amöben verschiedener Grösse und Gestalt (Fig. 1 b). Auf Gelatineplatten wuchs ein dem Proteus vulgaris äbnelnder Bacillus, jedoch wurde leider das weitere Studium dieses Mikroorganismus nicht verfolgt.

Die mikroskopische Untersuchung der Leberstücke liess fortgeschrittene Vernarbung der Abscesswandungen nachweisen. Die Membrana pyogenica bietet $z$ wischen der Detrituszone und embryonalem Gewebe viele Zooglöamassen, welche in ihren Einzelheiten aus dünnen spirillenartigen Gebilden bestehen. Im Abscess der Bauchmuskeln lassen sich ausser den in Leberabscess befindlichen Zooglöamassen auch einige Amöben in der Detrituszone nachweisen.

Fall 4. 33jähriger Fellah, türkischer Abkunft, ist am 22. September 1888 im Hospital aufgenommen. Patient hat am rechten Rippenbogen eine Fistel, die dickflüssigen rotbgefärbten Fiter secernirt. Anamnestisch erfahren wir, dass unser Kranker vor 3 Wochen an vorübergehenden dysenterischen Symptomen erkiankte mit gleichzeitiger schmerzhafter Anschwellung der Leber. Ein praktischer Arzt öfnete den Leberabscess mit dem Messer. Da die Wunde nicht rasch heilte, kam Patient in's Hospital. Die Fistel wurde gleich erweitert und der Finger ging in eine faustgrosse Höble. Dieselbe wurde ausgewaschen und drainirt. Patient erholte sich nicht mehr nach der Ausleerung des Abscesses, bekam profuse Diarrboe nach einigen Tagen und starb am 23. October.

Die Obduction hat das Diaphragua und Glisson'sche Kapsel sebr verdickt und verwachsen gezeigt. Auf der Oberfläche der Leber zwischen beiden 
Lappen befindet sich ein Sack durch Verwachsungen des Diaphragmas mit dem Lig. suspensor. gebildet. Der Sack erweist sich geöffnet als der operirte Leberabscess, der etwa $1 \mathrm{~cm}$ tief in die Lebersubstanz eingreift. Abscesswandungen narbig. Die Leber selbst vergrössert, blutreich und brüchig. Leber und Darmschlingen verkleben sich durch sulziges grünlich aussehendes Exsudat. Das Diaphragma ist glejchfalls mit der linken Lunge verwachsen. Letztere ist luftleer, graugrünlich aussehend. Die Bronchien sind wit sulzigem grünlich gefärbtem Exsudat angefüllt. Rechte Lunge unverändert.

Đie Schleimbaut des ganzen Darmtractus überall gelockert. Nan findet aber nirgends Geschwüre.

Die anderen Organe waren nicht besonders verändert. Die mikroskopische Untersuchung des Eiters hier war von keinem Belang, da der Abscess seit längerer Zeit mit der äusseren Luft in Verbindung war.

In den Schuitten der Leberwandungen, besonders in denjenigen, wo die Vernarbung nicht fortgeschritten war, fanden sich noch viele Amöben. Nebenan waren kleine Gruppen von Mikrokokken nachweisbar.

Fall 5. (Sefalis) Grieche, 15 Jahre alt, befindet sich seit 3 Monaten in Aegypten. Einige Wochen nach seiner Ankunft in Alexandrien erkrankt Patient an Dysenterie, welche nach 14tägiger Daver verschwand. Gleich darauf deutliche Symptome einer Lebervereiterung. Allmählich entwickelt sicb durch Schüttelfröste Fieber und rasche Abmagerung im rechten Hypochondrium ein orangengrosser Tumor. Patient war bei der Aufnabme sehr elend. Die Geschwulst erweist sich durch Punction als ein Leberabscess. Tod am 2. Tage nach der Punction.

Obduction. Leber gross. Am unteren Rand des linken Lappens ein faustgrosser Abscess. Die Kapsel des Abscesses ist bis $5 \mathrm{~cm}$ tief vernarbt. Im rechten Leberlappen befindet sich ein zweiter apfelgrosser Eiterheerd. Im Spigel'schen Lappen ein dritter eben so grosser Abscess, der diesen Lappen theilweise zerstört hat. Eiter rahmig.

Magen und Dünndarm unverändert. Im Dickdarm mehrere oberfächliche Erosionen (keine richtigen Geschwüre) vorbanden. Milz gross. Die übrigen Organe nicht verändert.

Sowobl im Darminhalt als auch in dem aus den zwei nichtoperirten Leberabscessen entnommenen Eiter sind viele lebende Amöben vorhanden. Aussaaten des Eiters ergeben keine Mikrobencolonien.

In den Schnittpräparaten der Abscesswandungen fanden sich viele Amöben, vorzugsweise in den Capillaren eingeschlossen. Ausser den Amöben keine Bakterien vorhanden.

Fall 6. (Metylen) Patient ist in's Hospital nach einer schweren Dysenterie eingetreten. Leber stark vergrössert, bei Druck schmerzhaft. Fieber und profuse Schweisse des Abends. Probepunction. Leberabscess. Operation desselben durch Resection der 10. Rippe. Orangengrosser Abscess. Tod.

Bei der Autopsie fanden sich, abgesehen von den operirten Abscessen des rechten Leberlappens, noch mnltiple Eiterheerde überall im Leberparen- 
chym. Der operirte Abscess war theilweise vernarbt. Dickdarm weist viele dysenterische Geschwüre auf.

Im Darmschleim zahlreiche Amöben, glejchfalls im Leberabscessinhalt. Des letzteren Aussaaten gaben schöne Colonien von Stapbylococcus a ureus. An Schnitten von den Darmgeschwüren siebt man im Geschwürsgrunde viele $A m o ̈ b e n$. In den Wandungen der Leberabscesse erkennt man bakterienfreie Amöben. Zwischen der Detrituszone der in Spindelform verwandelten Zellenreiben findet man dieselben isolirt. Hier liegen viele Mikrokokkencolonien in geschlängelten Capillaren eingeschlossen.

Fall 7. Kyprier I. Seit 3 Monaten Dysenterie, seit 4 Wochen deutliche Symptome von Leberabscess. Eröffnung des Abscesses durch Resection der 7. Rippe. Tod.

Ein Abscess im rechten Leberlappen von Kindskopfgrösse. Dickiarmgeschwüre. Leberabscesseiter auf Gelatine, Agar-Agar und Blutserum cultivirt, gab keine Colonien. Viele Amöben in den Darmgescbwüren, dieselben sind von stäbchenähnlichen Gebilden gefüllt. In den Leberabscesspräparaten fanden sich Amöben, sowie auch Mikrokokkencolonien, wie im Fall 6.

Fall 8. D. K., 40 Jahre alt, seit mebreren Monaten im Sudau (Dongola) beschäftigt, erkrankte 4 Wochen vor seiner Aufnahme in's Hospital an Dysenterie. Die blutigschleimigen Ausleerungen bestehen noch und zeigen mikroskopisch untersucht viele lebende Amöben. Gleichzeitig ist die Leber nach unten bedeutend vergrössert und auf Druck schmerzhaft. Tod nach Erschöpfung. Die Section hat überall viele tiefe Geschwüre des Dickdarmes gezeigt. Die Leber war stark vergrössert, sebr blutreich. Es fand sich im linken Lappen ein häbnereigrosser Abscess von dickflüssigem Eiter gefüllt.

Sowohl in den Darmschnitten als auch in denjenigen von den Leberwandungen fanden sich die Amöben wieder.

Fall 9. Ein 36jähriger Sudanese erkrankte 3 Monate vor seiner Aufnahme in's Hospital an Dysenterie. Patient ist sebr abgemagert, bat noeb blutschleimige Stühle und im rechten Hypochondrium einen kindskopforossen Tumor. Auf Probepunction und darauf erfolgter Eröffnung mit dem Messer fliesst schmutziger und übelriechender Eiter heraus. Tod.

Obduction. Leber gross, blutreich, von weicher Consistenz. Im rechten Lappen eine grosse Abscesshöhle, ihre Wandungen sphacelirt. Nebenbei findet man mehrere andere kleine Abscesse, sowie nekrotische Inseln auf der Oberfläche des Organs. Der grössere Abscess führt durch fistelförmige Wege in die Bauchmaskeln und erzengt daselbst einen walnussgrossen Eiterheerd.

Milz klein, derb. Nieren parenchymatös infiltrirt.

Der ganze Dickdarm ist derartig verschwärt, dass keine mehr umschriebenen Geschwüre unterscheidbar sind.

Lungen trocken, anämisch. Herz unverändert. Abscessinhalt enthält viele Amöben. Aus demselben züchtete ich zweierlei Mikroorganismen, einen 
Micrococcus, der nicht weiter verfolgt wurde, und einen Bacillus, der sofort als Bacillus pyogenes foetidus (Passet) erkannt wurde.

In den Darmschnitten Amöben in grosser Anzahl, sowie auch Massen von Bakterien. In den Abscesswandungen besteht die Detrituszone aus Micrococcuscolonien. Dieselben besitzen eine regelnässige Form, sind kegelförmig, geschlängelt, rund oder oval und machen den Eindruck von mikrokokkenhaltigen Amöben. Jedoch findet man neben ihnen deutliche mikrobenfreie Amöben von runder Gestalt, die weit bis in die Leberbalken eindringen.

Ein merkwärdiges Bild bieten die Muskelabscessschnitte. Hier findet man in der Detrituszone Haufen von kleinen Stäbchen zwischen Amöben, während inmitten des anscheinend intacten Muskelgewebes viele Capillaren von Mikrokokken angefüllt sind.

Fall 10. M., Araber, 40 Jahre alt. Dysenterie. Patient verschied einige Stunden nach seiner Aufnahme. Der Sectionsbefund, der 14 Stunden nach dem Tode vorgenommen wurde, bot Folgendes:

Vollständiger Schwund des Fettpolsters. Hautödem in den Füssen. Bei der Eröffnung der Bauchböhle zeigt sich die Leber stark vergrössert. Kleine linsengrosse Eiterheerde auf der Oberfläche des Organs. Die Leber braunroth, sehr blutreich und hart. Bei der Schnittführung findet man mehrere multiple Abscesse, deren grösserer kaum eine Haselnussgrösse erreicht. Auf der unteren Fläche der Leber befinden sich ähnliche Eiterheerde.

Milz mässig gross, nicht verändert.

Herz, Lungen, Nieren und Pankreas bieten niehts Auffallendes.

Magen etwas erweitert. Dünndarm anämisch. Der Dickdarm bietet in seiner ganzen Länge ein mannichfaltiges und buntes Aussehen. Die Scbleinbaut ist mit inselförmigen diphtheritisch aussehenden Ablagerungen, die einige Millimeter das Niveau überragen, versehen. Mit dem Messerrücken abgezogen, wird auch die Schleimhaut beschädigt. Viele Erosionen, überall der Schleimhant, deuten auf abgestossene diphtheritische Ablagerungen. Uebrigens gewahrt man isolirte runde oder irreguläre Geschwüre. Die diphtheritischen Ablagerungen erwiesen sich als nekrotisches Gewebe; unterhalb desselben, sowie in den richtigen Geschwüren, viele Amöben. Der Eiter enthielt todte Amöben, die Eiterkörperchen waren zerstört. Es wachsen daraus keine Colonien auf Gelatineplatten. In den Leberabscesschen fanden sich zahlreiche schönfarbene A möben, jedoch keine Bakterien.

Fall 12. G. K., 28 Jahre alt, aus Rhodos. Patient erkrankte vor einem Jahr angeblich zum ersten Mal an Malaria. Nach 40tägiger Krankheit und Darreichung von grossen Chiningaben wurde er wieder arbeitsfähig. Drei Monate vor seiner Aufnahme in's Hospital fing er wieder an zu fiebern, von Dysenterie soll er nichts bemerkt haben. Dann und wann verspürte er ziehende Schmerzen im rechten Schulterblatt und beim Tiefathmen war ihm die Leber schmerzhaft. Chinin wirkte nicht mehr und Patient magerte allmählich ab. Die Leber war bedeutend vergrössert und schmerzhaft, während 
die Milz normal zu sein schien. Auf Probepunction im 8. Intercostalraum kam dünnfüssiger, säuerlich riechender Eiter heraus. Berstung des Abscesses in die rechte Pleura. Tod.

Die Section wurde nicht gestattet und nur einige Leberstäcke wurden berausgeschritten.

Der Abseesseiter war zu Detritus verwandelt (Charcot's Krystalle). Weder Amöben noch Bakterien wurden gefunden. Auf den Nährsubstraten wuchsen keine Colonien. Ganz anders gestaltete sicb die mikroskopische Untersuchung der Abscessstücke. An der Membrana pyogenica (Detrituszone und Zellenzone) viele Amöben, nicht gut färbbar, aber mit erkenntlichen Vacuolen und einem kleinen Kern versehen. Mikrokokkenbaufen finden sich in der Begrenzungszone.

Fall 23. Idiopathischer Leberabscess. A., Aegypter, 40 Jahre alt. Patient war nie ernst krank, insbesondere hat er nie an Dysenterie oder Diarrhoe gelitten. Alcoholica genoss er sebr selten und rässig. Fr weiss selbst keine Ursache seines gegenwärtigen Leidens anzugeben. Ohne Weiteres fing ihm seit etwa 8 Wochen die Leber an schmerzhaft zu werden; er achtete aber nicht besonders darauf, als vor 35 Tagen eine Gesehwulst in seiner rechten Seite sich bemerkbar machte. Gleichzeitig fieberte er und schwitzte stark. Bei der Aufnahme war Patient sehr abgemagert, der Bauch tympanitisch. Unter dem rechten Rippenrand merkt man einen kindskopfgrossen Tumor dentlich fluctuirend. Gleich nach einer Probepunction, die übelriechenden Eiter ergab, profuse Diarrhoe. Peritonitis und Tod.

Obduction. Eitrige Peritonitis nach Berstung des im rechten Leberlappen befindlicben Abscesses. Leberabscess selbst kindskopfgross. (Eiter mikroskopisch untersucht, besteht aus Detrituskörncben und Charcot'schen Krystallen. Weder Amöben noch Bakterien vorhanden. Die Culturen blieben steril.)

Im Magen nichts Besonderes. Der Darm, abgeseben von einem leichten Katarrh seiner Schleimbaut, weist keine Geschwüre auf. Gleichfalls waren die anderen Organe unverändert gefunden. In den Schnittpräparaten der Leberabscesswandungen fanden sich nur an der Begrenzungszone kleine Gruppen von Mikrokokken, jedoch keine Amöben.

Fall 26. Idiopathischer Leberabscess. Polizois, 38 Jahre alt. Patient seit 18 Monaten in Aegypten, soll angeblicb stets gesund gewesen sein, giebt aber an, durch Armuth ungeeignete Nahrungsmittel genossen zu haben. Seine Wohnung war ebenfalls schlecht und feucht. Secbs Tage vor seiner Aufnahme in's Hospital verspürte er plötzlich einen stechenden Schmerz in seiner Lebergegend, darauf bohes Fieber und profuses Schwitzen. Bei der Aufnahme war die Leber bedeutend vergrössert und auf Druck sehmerzbaft, die Abendtemperatur $40^{\circ}$. Eröffnung des Abscesses durch Resection der 8 . Rippe. Durchbruch eines zweiten Abscesses in die rechte Pleurahöhle und Tod.

Die Obduction bestätigte, dass ausser dem operirten Abscess ein zweiter im Oberrand des rechten Leberlappens befindlicher Abṣcess durch das Dia- 
phragma in die rechte Plenra durchgebrochen war. Die Pleura selbst entbält eine grosse Menge vou Eiter und ist in ibren Wandungen mit Pseudomembranen bekleidet. Der operirte L Leberabscess un die Hälfte vernarbt.

Milz gross, ibre Schnittfäche weist mehrere bämorrhagische Heerde auf.

Magen unverändert. Die Schleimbaut des D ünndarms etwas gelockert, die Peyer'schen Plaques breiter und deutlicher als gewöhnlich. Im Dickdarm findet man auf der Klappe 3-4 kleine Brosionen der Sebleimhaut. Im Wurmfortsatz befindel sich ein Olivenkern. Die Schleimhaut stark entzündet und theilweise verschwärt. Im übrigen Dickdarm sieht man keine anderen Verä日derungen. Von den übrigen Organen waren noch beide Nieren vergrössert und leicht entzündet.

Schnitte vom Wurmfortsatz zeigen in der Submucosa die Gefässe erweitert. Einige derselben enthalten Mikrokokkencolonien.

In den Schnitten des nicht operirten Leberabseesses waren schöne Colonien von Mikrokokben zu sehen.

Die übrigen von mir untersuchten Leberabscesse lasse ich, der Kürze wegen, in amstehender Tabelle folgen.

Mehrere andere Leberabscesse, die entweder aus der Privatpraxis stammten, oder deren Eiter mir von befreundeten Collegen zur Verfügung gestellt wurde, ergaben ganz ähnliche Verhältnisse. Bei Leberabscessen dysenterischen Ursprangs sind die A möben, wie schon bemerkt, wenn auch nicht immer lebensfähig, doch im Eiter von alten Abscessen vorhanden, während die Bakterien in der abgeschlossenen Abscesshöhle, wie es scheint, schnell zu Grunde gehen. Dafür spricht der Umstand, dass in den meisten Abscessen, besonders in denjenigen, die seit längerer Zeit bestanden, der zu Culturen verwandte Eiter nur in sehr wenigen Fällen keimfähig war. Andererseits aber fanden sich in den Gefässen oder im Detritus der Abscesswandungen Bakterien. Es ist deshalb sehr interessant, in der Zukunft den Eiter von ganz frischen Leberabscessen durch Cultur genau zu erforschen. Dann kann man auch nạchträgliches Eindringen von fremden Mikroorganismen ausschliessen. Ich halte es für möglich, dass die $2 \mathrm{mal}$ von mir cultivirten Bacillen [Bacillus foetidus und Proteus vulgaris (?)] ursprünglich nichts mit der Genese des Abscesses zu thun hatten und dass vielmehr nur Mikrokokken die Lebereiterung hervorbringen. Das gilt besonders für diejenigen Formen des Leberabscesses, welche ich als idiopathisch angefïhrt habe. Hier waren nur Mikrokokken, und zwar die bekannten Staphylokokken aureus und albus 
Dysenterische Leberabscesse.

\begin{tabular}{|c|c|c|c|c|c|c|}
\hline & Fall. & $\begin{array}{c}\text { Gebalt } \\
\text { des Eiters } \\
\text { an } \\
\text { Amöben. }\end{array}$ & $\begin{array}{l}\text { Gehalt des } \\
\text { Eiters an } \\
\text { Bakterien. }\end{array}$ & $\begin{array}{c}\text { Culturales } \\
\text { Verhalten des } \\
\text { Eiters. } \\
\text { Bakterien. }\end{array}$ & $\begin{array}{c}\text { Verhalten der } \\
\text { Schnitte. }\end{array}$ & Bérnerkungen. \\
\hline 1. & M. Ibrahim & Amöben & nichts & nichts & nur Awöben & - \\
\hline 2. & Jude J. & nichts & $"$ & 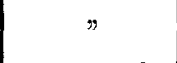 & m & $\begin{array}{c}\text { Eiterkörperchen zer- } \\
\text { stört. }\end{array}$ \\
\hline 3. & Kretenser & Amöben & ” & Proteus (?) & $\begin{array}{c}\text { Amöben und } \\
\text { Bacillen }\end{array}$ & - \\
\hline 4. & türk. Fellah & nichts & $"$ & nichts & $\begin{array}{l}\text { Amöben und } \\
\text { Nikrokokken }\end{array}$ & $\begin{array}{l}\text { Abscess meisten- } \\
\text { theils vernarbt. }\end{array}$ \\
\hline 5. & Sefalis & Amöben & & - & nur Amöben & - \\
\hline 6. & Metylen & $"$ & Mikrokokken & $\begin{array}{l}\text { Staphylococ } \\
\text { eus aureus }\end{array}$ & $\begin{array}{l}\text { Amöben und } \\
\text { Mikrokokken }\end{array}$ & - \\
\hline 7. & Cyprier I & $n$ & nichts & nichts & $"$ & $\therefore \quad-$ \\
\hline 8. & D. Kalo & $"$ & $\Rightarrow$ & - & nur Amöben & $\begin{array}{l}\text { keine Culturen an- } \\
\text { gesetzt. }\end{array}$ \\
\hline 9. & Sudanese & $"$ & $\begin{array}{l}\text { kleine } \mathrm{Ba}- \\
\text { cillen }\end{array}$ & $\begin{array}{c}\text { Bacillus foe- } \\
\text { tidus }\end{array}$ & $\begin{array}{c}\text { Amöben und } \\
\text { Bacillen }\end{array}$ & - \\
\hline 10. & M., Araber & & nichts & nichts & nur Amöben & - \\
\hline 11. & Rbodius & nichts & $m$ & $\Rightarrow$ & $\begin{array}{l}\text { Amöben und } \\
\text { Mikrokok ken }\end{array}$ & alter Abscess. \\
\hline 12. & G. K., Griecbe & & $"$ & & " & - \\
\hline 13. & Kephalon & Amöben & 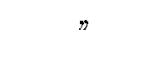 & $\begin{array}{l}\text { Staphylocoe- } \\
\text { cus albus }\end{array}$ & 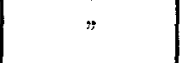 & - \\
\hline 14. & Cyprier II & $"$ & " & nichts & 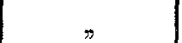 & - \\
\hline 15. & Trautus & - & 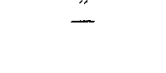 & - & $"$ & $\begin{array}{l}\text { nur Schnitte unter- } \\
\text { sucht. }\end{array}$ \\
\hline 16. & Metylen II & $=$ & - & - & 7 & . \\
\hline 17. & Older & Amöben & nichts & $\begin{array}{c}\text { Staphylococ- } \\
\text { cus aureus }\end{array}$ & 3 & nicht secirt. \\
\hline $\begin{array}{l}18 . \\
19 .\end{array}$ & S. $\frac{k y r}{x}$. & $"$ & 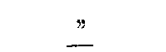 & nichts & A möben und & nur Leherstücke \\
\hline 20. & Fuyer & Amöben & nichts & - & Mikrokokken & $\begin{array}{l}\text { untersucbt. } \\
\text { nur der Eiter mikro- } \\
\text { skopisch. }\end{array}$ \\
\hline $\begin{array}{l}21 . \\
22 .\end{array}$ & $\begin{array}{c}\text { Meson } \\
\text { Cirkassier }\end{array}$ & " & 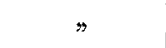 & - & - & $\eta$ \\
\hline & Curkassier & $"$ & $"$ & - & 一 & $"$ \\
\hline
\end{tabular}

Idiopathische Leberabscesse.

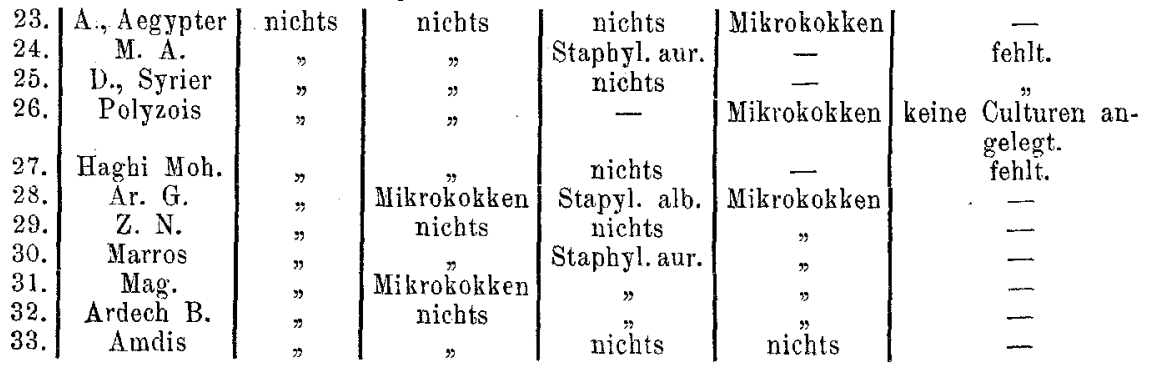




\section{8}

nachgewiesen. Anders verhält sich die Sache bei den dysenterischen Leberabscessen, wo alle möglichen Organismen durch das Darmgeschwär Eingang finden können. Hier waren stets die Amöben vorhanden, in einigen Fällen aber fanden sich Mikrokokken und Bacillen. Ob letztere die Eiterung in diesen Fällen erzeugen, lasse ich aus den oben mitgetheilten Gründen noch unentschieden.

Die pathologischen Veränderungen in den tropischen Leberabscessen sind je nach dem Alter des Abscesses verschieden. Gewöhnlich sitzen dieselben im rechten Leberlappen. Isolirte Leberabscesse sind im linken Lappen seltener, meistentheils kommen sie im letzterwähnten Lappen in multipler Form vor. Im Spigel'schen Lappen habe ich nie einen isolirten Leberabscess beobachtet. Die meisten solitären Leberabscesse liegen in der Mitte der Leber, auf der Oberfläche derselben sind sie seltener und sind dann wieder gewöhnlicher auf der vorderen als auf der hinteren Seite der Leber anzutreffen. Kleine Eiterhöhlen, sowie nekrotische Inseln sind meistens oberflächlich gelagert.

Betrachtet man einen Leberabscess näher, so findet man in den ganz frisch entstandenen Eiterhöhlen die Wandungen eben. Der Inhalt ist rahmig, hier und da mit Blut (was noch bei dysenterischen Leberabscessen besonders vorzukommen scheint) gemengt. Die sog. Membrana pyogenica ist noch nicht deutlich entwickelt und besteht aus zerfallenem Gewebe. In dem Detritus erkennt man hier und da noch einige Zellen und Schollengebilde, um die Capillaren eine enorme Ansammlung von Leukocyten, die auch bis zum Detritus fortgeschwemmt werden. Man erkennt an derartigen Eiterhöhlen im Allgemeinen, dass die $\mathrm{Mem-}$ brana pyogenica eigentlich aus drei Zonen besteht, welche aber nicht scharf in allen Fällen zu bestimmen sind. Meistentheils aber sieht man dieselben aus Detrituszone, Zellenzone und Begrenzungszone bestehend. Die Detrituszone wird aus kleinen runden, ovalen und unregelmässigen Körnchen gebildet. Eiterkörperchen findet man hier nicht. Oft aber wird die Grundsubstanz dieser Zone aus coagulirtem Fibrin gebildet und die Körnchen bedecken dieselbe theilweise oder ganz. Gallenpigment ist auch oft hier zu sehen. Die Körnchen neh- 
men die Anilinfarbe stark an, während das Fibrin schwach gefärbt wird. Bei dysenterischen Abscessen sind auch Amöben, wie oben schon bemerkt wurde, vorhanden. Bakterien jedoch werden hier selten gefunden. Nur in wenigen Fällen bemerkte ich, dass die Detrituszone anstatt von Körnern, von Zooglöamassen bedeckt war. Allmählich nacb innen geht man in die Zellenzone über. Es sind das vorwiegend junge Zellen, welche durch Farben stark tingirt werden. Einige Zellen von runder oder ovaler Form sind doppelt so gross, besitzen keinen deutlichen Kern und weisen ein feinkörniges Protoplasma auf. In Zwischenräumen findet man hier und da stark beschädigte Leberzellenreihen, isolirte Leberzellen selbst oder Capillargefässe. Oft aber ist die Anhäufung von jungen Zellen eine so grosse, dass dieselben einen richtigen Wall gegen die Begrenzungszone bilden. Letztere, die die Grenze zwischen dem erkrankten und dem intacten Lebergewebe bildet, besteht aus zusammengedrückten Leberzellen und jungen Gefässen. Um dieselben häufen sich Leukocyten, junge Bindegewebszellen und junge Gallengänge. In den Gefässen befinden sich entweder Bakterien oder Amöben. Merkwürdig ist in dieser Zone, deren Grenzen je nach der Intensität des Prozesses nicht näher zu bestimmen sind, eine spindelförmige Verwandlung der Zellenreihen. Offenbar geschieht dies durch entzündliche Prozesse, sowie auch durch den Druck, welcher von den erweiterten Gefässen, von den ausgewanderten Leukocyten und von dem ergossenen Blut auf dieselben ausgeübt wird. Die Leberzellenreihen verlieren ihren Zusammenhang und so wird allmählich jeder Zellenschlauch in eine Spindelzelle zusammengeschrumpft. Einige dieser Gebilde nehmen so eine faserige Gestalt an, man erkennt an ihnen einen kleinen Kern und es ist schwer, dieselben von ächtem Bindegewebe zu unterscheiden. Ein anderes Bild ist hier auffallend, nehmlich die neugebildeten Gallengänge. Dieselben treten zwischen dem embryonalen Gewebe auf, besitzen oft ein weites Lumen, sind länglich, oval, oder wellenförmig. Ihr Lumen ist stets leer, die Wandung aber von regelmässigem Endothel bekleidet. Bei fortgeschrittener Vernarbung in den Leberabscesswandungen ist die Neubildung von Gallengängen viel stärker. In der Begrenzungszone findet man übrigens viele Plasma- 
zellen, die uin so reichlicher auftreten, je weiter der Prozess der Vernarbung fortgeschritten ist. Die Vernarbung beginnt stets von innen nach aussen von den portalen Gefässen zuerst durch Ansammlung von jungen Zellen um dieselben, sowie durch Neubildung von jungen Gefässen und Gallengängen. Allmählich nimmt das Keimgewebe eine fasrige Gestalt an. Nach innen gestalten sich noch die Gefässe sehr erweitert, mitunter auch sind ihre Wandungen verdickt, und einige strotzen von Blut. Um dieselben findet eine kleinzellige Infiltration statt, welche bis in die Zwischenspalten der Gallenschläuche eindringt.

Dass die Leberzellenreihen, die spindelzellig sich verwandeln, endlich zu Bindegewebefasern führen, halte ich für sehr wahrscheinlich. Ich babe mehrmals an älteren Abscessen beobachtet, wie dieselben sich verlängern, verdünnen und von faserigen Bündeln nicht zu unterscheiden sind. Allmählich schreitet auf diese Weise der Vernarbungsprozess nach aussen fort und es bildet sich endlich eine feste Bindegewebskapsel oder auch wie bei kleineren Abscessen der Fall ist, ein faseriger Ring, wodurch die Eiterhöhle vom intacten Leberparenchym getrennt wird.

\section{Schluss.}

Aus dem Mitgetheilten ist zur Genüge ersichtlich, dass in Aegypten die Amöben eine ätiologische Rolle zur Genese der dysenterischen Leberabscesse spielen und dass pyogene Mikroorganismen es sind, wahrscheinlich aus dem Gastrointestinaltractus stammend, die die idiopathischen Leberabscesse hervorrufen.

Erst seit kurzer Zeit wurde die Aufmerksamkeit der Forscher auf die Protozoen oder Iponzoen gelenkt und in dieser Frist lernten wir einige Krankheiten, welche ihre Ursache diesen Parasiten verdanken. Ich erinnere nur an die Plasmodien von der Malaria, sowie an die Gregarinen (Pfeiffer). Eine Schwierigkeit bietet die Forschung dieser Thierchen, dass sie sich nicht so leicht wie die Bakterien züchten lassen. Gelingt dies einmal, so wird man auch den noch fehlenden Beweis des Thierversuches erbringen können. 


\title{
Erklärung der Abbildungen. \\ Tafel VIr.
}

Fig. 1. a Aufstrichpräparat. Eiter des Leberabscesses von Fall 3. Mit Carbolfuchsin gefärbt. Amöben verschiedener Grösse mit vielen Vacuolen. b Cultur der obigen Amöben im sterilen Bouillon-Gelatine-Wasser. III. Umzüchtung. Schwärmerform mit lebhaften Bewegungen und vielen Vacuolen. Gefärbt mit Carbolfuchsin. (Ocul. 3, Obj. CC. Zeiss.)

Fig. 2. Leberscbnitt aus dem Falle 5 (Sefulis). Ein Gefäss oit Fibrin und 3 vacuolenbaltigen Amöben. Leberzellenreiben gedrückt. In den $Z$ wischenspalten Blut. Nach Loeffler gefärbt. (Ocul. 3, Obj. CC. Zeiss.)

Fig. 3. Leberschnitt (Detrituszone) aus dem Abscess Fall 2. Finige Gefässe noch intact mit A möben. A möben befinden sich gleichfalls $z w i-$ schen der Detrituskörnchen. Nach Loeffler gefärbt. (Ocul. 3, Obj. CC. Zeiss.)

\section{IX.}

\section{Narkotische Wirkungen von Hydroxylamin und Natriumnitrit.}

\author{
Von Prof. C. Binz in Bonn.
}

Was ich bisher Experimentelles über Hydroxylamin und Natriumnitrit veröfentlicht habe, steht in Zusammenhang mit dem, was man therapeutisch und toxikologisch über einige $\mathrm{Ha}$ logenverbindungen weiss. Die Zahl der letzteren, welche lähmend auf die Nervencentren des Warmblüters wirken, ohne dass sie eine Kohlenstoffverbindung enthalten, ist in nevester Zeit vermehrt worden durch das Fluornatrium. Aus diesem Jahre liegen drei experimentelle Arbeiten darüber vor ${ }^{1}$ ). Andere ähnliche Körper habe ich früher als narkotisirend erwiesen und in Bd. 113 dieses Archivs S. 6 zusammengestellt und besprochen.

Das Fluornatrium bringt nach Tappeiner beim Frosch das ganze centrale Nervensystem, die Nervenfasern und die motorischen Endplatten zur Lähmung, und zwar direct, ohne weiteren

1) Siehe das Literaturverzeichniss am Schluss. 